\title{
Examining spatial disparities in electric vehicle charging station placements using machine learning
}

\author{
Avipsa Roy ${ }^{1,2 *}$, Mankin Law ${ }^{1}$ \\ ${ }^{1}$ Department of Urban Planning and Public Policy, University of California, Irvine \\ ${ }^{2}$ Institute of Transportation Studies, University of California, Irvine
}

*Corresponding Author:

Avipsa Roy, Ph.D.

Assistant Professor

Department of Urban Planning and Public Policy

School of Social Ecology

University of California, Irvine

Address: 300 Social Ecology I

Irvine, CA 92697

Email: avipsar@uci.edu 


\section{Abstract}

Electric vehicles (EV) are an emerging mode of transportation that has the potential to reshape the transportation sector by significantly reducing carbon emissions thereby promoting a cleaner environment and pushing the boundaries of climate progress. Nevertheless, there remain significant hurdles to the widespread adoption of electric vehicles in the United States ranging from the high cost of EVs to the inequitable placement of EV charging stations (EVCS). A deeper understanding of the underlying complex interactions of social, economic, and demographic factors which may lead to such emerging disparities in EVCS placements is, therefore, necessary to mitigate accessibility issues and improve EV usage among people of all ages and abilities. In this study, we develop a machine learning framework to examine spatial disparities in EVCS placements by using a predictive approach. We first identify the essential socioeconomic factors that may contribute to spatial disparities in EVCS access. Second, using these factors along with ground truth data from existing EVCS placements we predict future ECVS density at multiple spatial scales using machine learning algorithms and compare their predictive accuracy to identify the most optimal spatial resolution for our predictions. Finally, we compare the most accurately predicted EVCS placement density with a spatial inequity indicator to quantify how equitably these placements would be for Orange County, California. Our method achieved the highest predictive accuracy $(94.9 \%)$ of EVCS placement density at a spatial resolution of $3 \mathrm{~km}$ using Random Forests. Our results indicate that a total of $74.18 \%$ of predicted EVCS placements in Orange County will lie within a low spatial equity zone - indicating populations with the lowest accessibility may require the highest investments in EVCS placements. Within the low spatial equity areas, $14.86 \%$ of the area will have a low density of predicted EVCS placements, $50.32 \%$ will have a medium density of predicted EVCS placement, and only 9\% tend to have high EVCS 
placements. The findings from this study highlight a generalizable framework to quantify inequities in EVCS placements that will enable policymakers to identify underserved communities and facilitate targeted infrastructure investments for widespread EV usage and adoption for all.

Keywords: Electric vehicles, spatial equity, machine learning, scale

\section{Introduction}

Adoption of emerging alternative transportation modes like EVs has been identified as a primary requirement to meet California's climate mitigation goal (Williams et al., 2012). EVs provide the opportunity to reduce localized air pollution (Brady \& O’Mahony, 2011) as well as reduce traffic noise (Hawkins et al., 2013) in cities. As part of the U.S government's EV charging action plan (White House, EV Charging Action Plan, 2021), the state of California and many of its cities have aggressive goals for achieving high EV penetration by 2030. For example, the City of Los Angeles aims to have $80 \%$ of its vehicle fleet electrified by 2035 (City of Los Angeles, 2019). The City of San Francisco has a 2030 goal to make EVs 100\% of all new vehicle sales (San Francisco Electric Vehicle Working Group, 2019). However, California currently has less than $40 \%$ of the charging infrastructure needed by 2025 to support the projected EV fleet (Nicholas et al., 2019). The steps taken to deploy charging infrastructure by local authorities will therefore tend to determine who would benefit most from such investments.

Access to current EV charging stations is extremely inequitable throughout the United States, leaving large percentages of the population without affordable access to electric vehicles necessary for employment opportunities and access to essential goods and services. Low-income populations, which comprise people of color and people with disabilities suffer from the largest 
barriers to access (Marcantonio et al. 2017) and are therefore disproportionately vulnerable to EV adoption. Some of the primary barriers to extensive electric vehicle (EV) adoption in urban areas are the high purchase cost (Adepetu and Keshav, 2017), the vehicle travel range which often leads to range anxiety among drivers, and the lack of sufficient amount of charging infrastructure availability (Bakker and Jacob Trip, 2013; Egbue and Long, 2012; She et al., 2017) distributed uniformly across an entire geographic region (E.g.: country/state/city). The socioeconomic barriers have limited the demographics of early and current EV owners with populations from higherincome neighborhoods, with high levels of education and living in single-family households (California Clean Vehicle Rebate Project, 2015; Carley et al., 2013; Farkas et al., 2018). Some studies have shown that lower-income or minority communities are still lagging in terms of access to EV usage due to cost barriers, lower availability of the technology (Dailey et al., 2010; Judge et al., 2004 Brockway et al., 2021), and fewer programs facilitating technology uptake (Warschauer et al., 2004). However, further research is needed to identify the explicit factors contributing to spatial inequities in EVCS access across different cities as they are still not very well understood by planners and policymakers.

Factors like purchase cost and battery range are similar across markets and present the overall barriers to EV usage and mass adoption across a larger scale (Hsu \& Fingerman, 2021). Some studies have used machine learning algorithms to coordinate EV chargers (Shibl et. Al., 2020) while others have looked at profit-maximization approaches to EVCS placements (Huang and Kockelman, 2020) and the charging demand of EV users from public charging stations based on previous charging behavior (Almaghrebi et al., 2020). However, these studies did not investigate the differential access of public charging infrastructure between different sociodemographic groups at finer spatial scales. 
More recently, U.S. cities are focusing on equity issues related to EV and charger access (Evolve Houston, 2019; San Francisco Electric Vehicle Working Group, 2019). To our knowledge, no extensive systematic analyses have yet been considered on how to equitably place EVCS in cities based on their underlying socioeconomic characteristics. Addressing such equity issues related to EV charging stations has been limited owing to a lack of good quality data. Previously, solar panel data (Sunter et al., 2019) have been used to understand charging availability in disadvantaged communities (Canepa et al., 2019), but further research is needed to examine spatial disparities in EVCS placements and access.

In this study, we aim to answer the following research questions around equitable EVCS placements: (a) What are the social, economic, and demographic factors that can quantify spatial inequity in EVCS access? (b) Which is the most appropriate spatial scale at which EVCS placements can be predicted accurately after accounting for these factors? (c) How would future EVCS placements distribution vary spatially with existing inequities in place? The overall spatial distribution and availability of charging infrastructure can highlight the inequities in EV usage at smaller scales depending upon the frequency and densities of local deployment.

Our study aims to bridge this gap using a machine learning approach. By combining disparate data sources from EV charging stations and solar panels (which act as a proxy for EV usage) along with social, economic, and demographic factors this study highlights existing disparities and barriers to access to EV charging infrastructure. Machine learning algorithms are then used to generate predictive maps of EVCS placements which are compared with different levels of spatial inequity to quantify the accessibility issues related to these EVCS placements.

The method developed in this study is open and reproducible and can be used in other counties and states for conducting similar analyses. The results generated from this study will enable 
policymakers to make informed decisions by visually examining the spatial distributions of EV charging infrastructure and identifying areas that may need more targeted infrastructure planning and management in the future to ensure equitable access among all populations.

\section{Data \& Study Area}

We selected Orange County in Southern California as our study area to understand the spatial inequities of EV charging access. Orange County is one of the highest density regions in the country in terms of EV ownership and charging infrastructures in the state of California (California Energy Commission, 2022). The county is immensely heterogeneous in terms of income and population characteristics (Figure 1), thereby making it an ideal place to study EV charging access and equity issues.

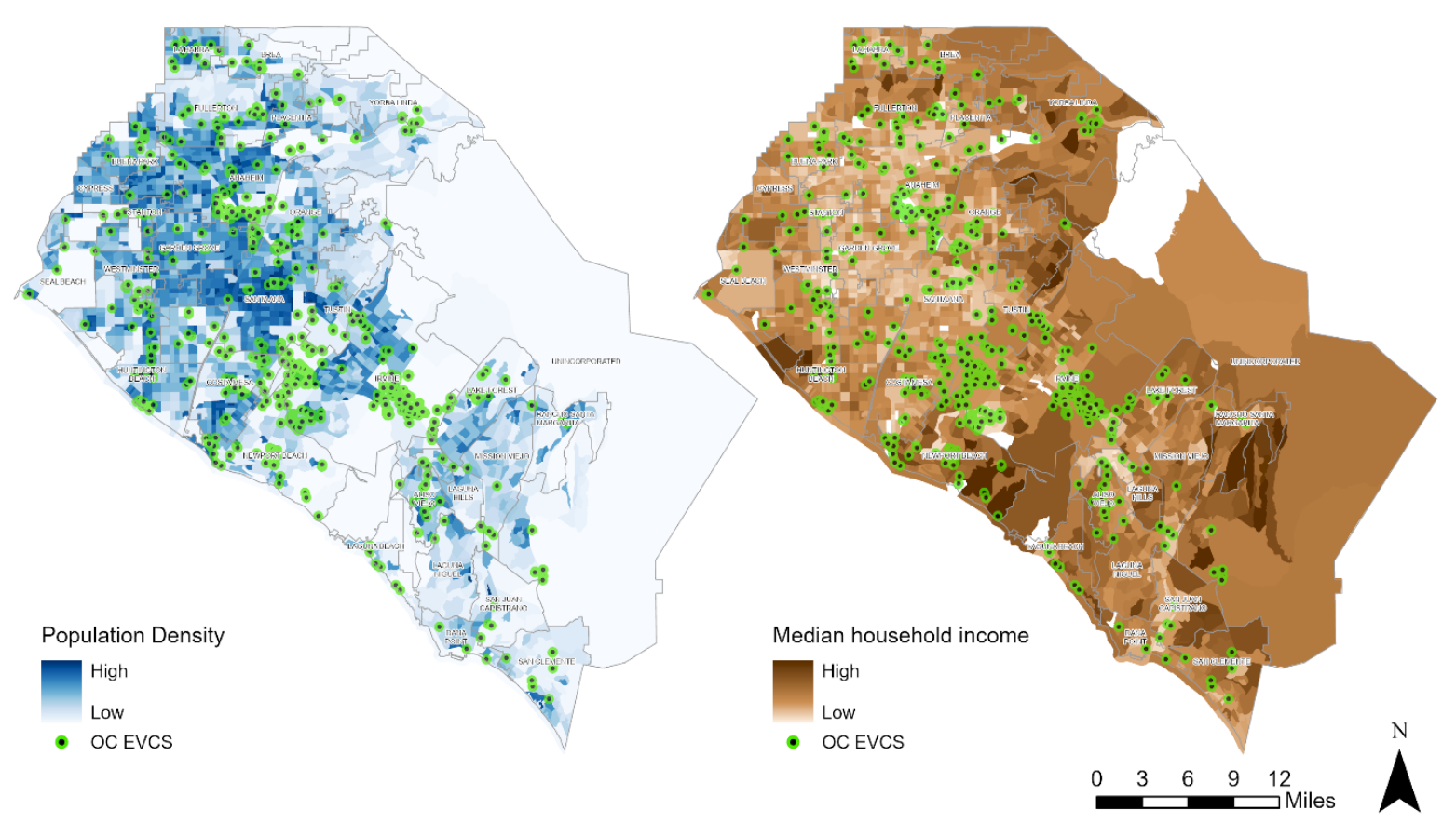

Figure 1: Spatial distribution of existing EV charging stations across Orange County w.r.t to (a) population density and (b) median household income distribution 
As of 2021, the county has over 78,000 registered EVs with only 5,852 existing public and private EVCS (Table 1), therefore, the current service coverage of existing EVCS is worth investigating as well as crucial for future infrastructure planning in this highly car-dependent county.

The central part of the county has the highest discrepancies in terms of population density and income levels (Figure 1) with cities like Santa Ana, Orange, Garden Grove, Tustin and Costa Mesa. Also in the southern part of the county cities like Laguna Niguel, San Clemente, and San Juan Capistrano are mostly underserved where the coverage of EVCS is much lower compared to population density. However, the comparatively wealthier neighborhoods near Irvine, Newport Beach and some parts of Huntington Beach have higher numbers of existing charging stations well distributed throughout the cities. The data applied in our models and analysis are collected from open data portals provided by the Orange County, California Energy Commission, Department of Energy, and the U.S. Census Bureau (Table 1).

Table 1: Summary of the socioeconomic statistics in Orange County

\begin{tabular}{lc}
\hline Socioeconomic characteristics & Estimate** \\
\hline Total Population & $3,175,692$ \\
Median Age & 38.6 \\
Median Household Income & $\$ 90,234$ \\
Median Home Value (Owner-occupied) & $\$ 679,300$ \\
Persons in poverty (Percent) & $9.5 \%$ \\
Mean Travel Time to Work (Workers age 16 years+) & 28 minutes \\
Zero Emission Vehicle Population (as of end of 2020) & 78,256 \\
Cumulative Battery Electric Vehicle Sales (as of 2021 Q3) & 73,046 \\
Total Number of EV Chargers (Public and shared private Lv1, Lv2, and DC Fast) & 5,657 \\
\hline Source: 2019 ACS 5-year estimates, California Energy Commission &
\end{tabular}




\section{Methods}

The overall methodology of this study combines machine learning along with quantitative spatial analysis as described in the workflow diagram in Figure 2. We first evaluated the existing EVCS placements using kernel density estimation (KDE) and classified three levels of intensities based of the KDE results. Then, we extracted relevant variables from chosen sources and spatially joined the features into different sizes of the fishnet grids . Using the extracted variables and KDE classes, the next step is to apply machine learning model fitting to predict the EVCS grids.

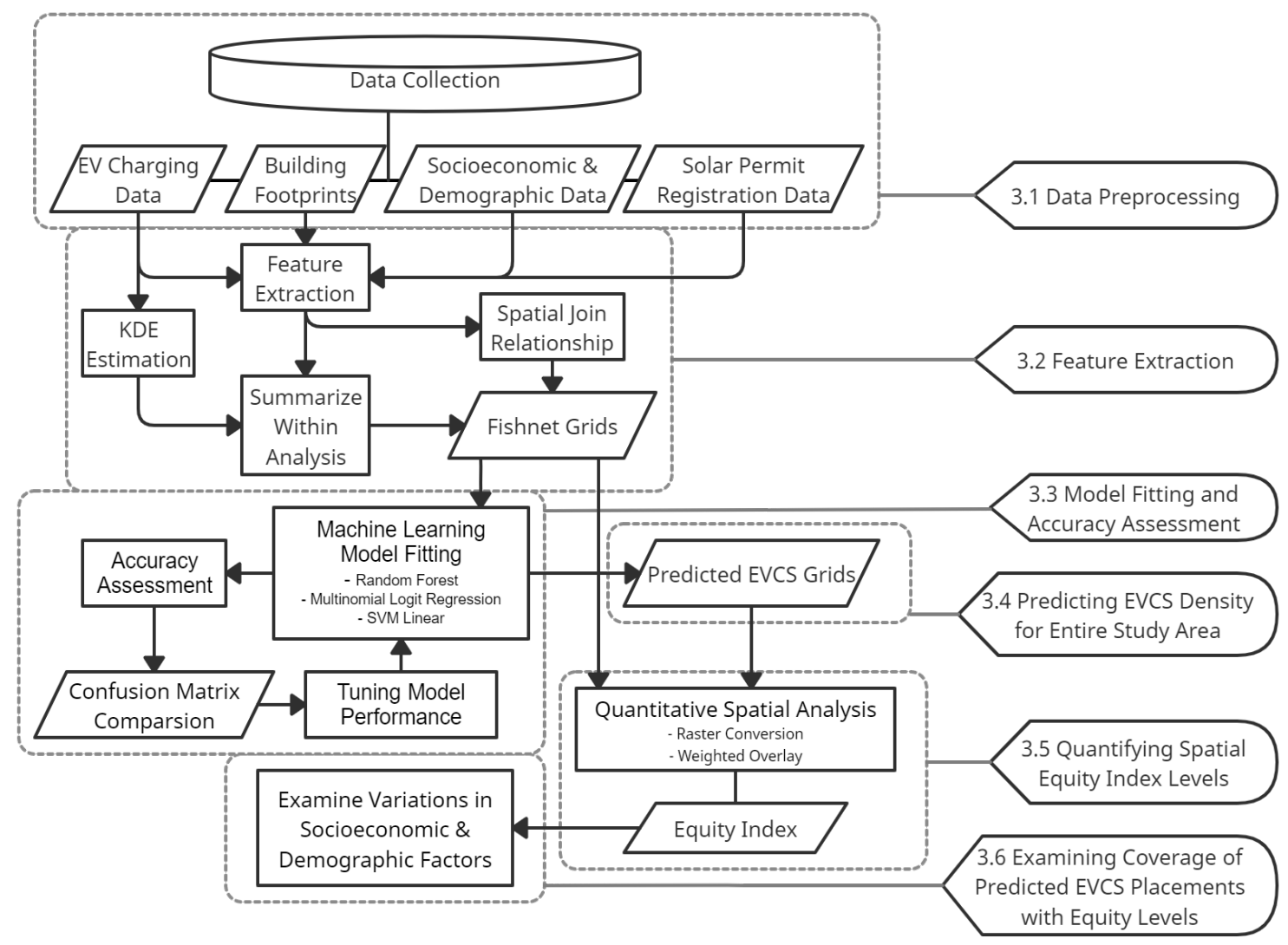

Figure 2: Spatial distribution of existing EV charging stations across Orange County w.r.t to (a) population density and (b) median household income distribution 
This procedure includes testing the model accuracy using different training methods, confusion matrix comparison, and tuning the model performance repeatedly. After that, the model with the highest accuracy will then be summarized into our spatial analysis with other relevant features to determine whether communities have equitable access to EVCS. Lastly, we develop an equity index using a raster weighted overlay to demonstrate the access disparities and compare them with current infrastructure coverage. Each step is discussed elaborately in subsections 3.1 through 3.6.

\subsection{Data preprocessing}

In the data preparation phase, we combined data on existing EVCS, socioeconomic characteristics of neighborhoods at the census block group level and information on housing density as well as summarize the spatial relationship among the collected datasets. These variables were meaningfully chosen for quantifying EVCS access based on previous work (Shahriar, 2021; Law \& Roy, 2021), and preprocessed in ArcGIS and converted to raster format ensuring the spatial resolution of all data layers were matched. We addressed multicollinearity by ensuring the Pearson's correlation coefficient among all variables were below 0.4 . The feature classes are listed in Table 2 and demonstrate the open data resources used for this analysis. The EVCS locations collected from several sources were converted to a raster format using kernel density estimation (KDE) for matching it with the other data layers and smoothing out the density distributions. The KDE results were then categorized into three levels high, medium and low using a natural Jenks classification. These categories of EVCS density were used as ground truth for training the machine learning models. 


\subsection{Feature extraction from independent variables}

We divided our study area into grids to better represent the spatial distribution of EV charging infrastructures and associated the relevant variables from Section 3.1 to each grid for a joint investigation of the spatial variations in socioeconomic attributes along with EVCS distributions. Using the fishnet tool in ArcGIS, we divided the study area into spatial grids. We varied the scale of our analysis using different grid sizes ranging from $1 \times 1 \mathrm{~km}$ to $5 \times 5 \mathrm{~km}$ to estimate the prediction accuracies of the models at multiple scales. The preprocessed data from socioeconomic and built environment variables extracted in Section 3.1 were then spatially joined to the grid features for all grid sizes. Finally, the summary statistics of the extracted features for each grid cell were calculated using mean and standard deviation from the joined dataset. We repeated this process by varying the grid sizes from $1 \mathrm{~km}$ up to $5 \mathrm{~km}$.

\subsection{Model fitting and accuracy assessment}

Our machine learning model predicts the EVCS grid density levels within Orange County. We used the caret (Classification And Regression Training) ' $R$ ' package to train three different machine learning models by varying the spatial grid sizes from $1 \mathrm{~km}$ to $5 \mathrm{~km}$. The algorithms used for training the three different models were - random forests (rf), multinomial logistic regression (mlr), and support vector machines (svm). We used $80 \%$ of the data for training our models and used the held out $20 \%$ of the data for testing the accuracy of the models. To ensure the training data are randomized and the models do not overfit, we implemented a control mechanism called repeated ' $\mathrm{k}$ ' fold cross-validation, such that the training data were split into ten folds and models were repeatedly fitted in each fold with a new dataset. The process was repeated three times for 
each algorithm. Eventually, we generated a total of 15 models which were compared among each other for the best fit by comparing the accuracies of each model with a total of 30 iterations.

\subsection{Predicting EVCS placements for entire Orange County}

After model training, we computed the mean cross-validation accuracy among three algorithms and five grid sizes to determine the most accurate model for prediction among all fifteen models. Based on the training data, we also calculated the precision, recall, and F1-scores for each model. The most accurate model was then used to predict EVCS density classes from the test data. The predicted and actual class labels were also used to generate confusion matrices that highlighted how accurately each EVCS density class was predicted at different spatial scales. Finally, we selected the model with the highest cross-validated accuracy, maximum F1-score and lowest misclassification rates to predict the EVCS placement density classes for the entire Orange County at the most appropriate spatial grid size. A map of the predicted EVCS placements at the chosen spatial scale was then generated for the entire study area.

\subsection{Quantifying spatial equity levels for EVCS placements}

Since the key objective of this study is to understand equity access of EVCS placements, we computed a weighted equity index to demonstrate the spatial variations in overall accessibility of the predicted EVCS placements. Raster layers constructed from the socioeconomic and demographic features listed in Table 2 and were used to generate a weighted index called the Electric Vehicle Charging Inequity (EVCI) index using a multicriteria decision analysis approach developed by Roy \& Kar (Roy \& Kar, 2022). The outcome was a weighted equity index map (at 
the chosen spatial resolution from section 3.4) was generated to highlight the levels of spatial equity throughout Orange County for the spatial grid size chosen in Section 3.4.

\subsection{Quantifying spatial equity levels for EVCS placements}

The weighted index map generated in Section 3.5 was overlaid with the predicted EVCS placements map to visually compare how equitably the future EVCS would be placed. The spatial coverage of the EVCS placements was computed from the percentage of area covered by the predicted EVCS placements that overlapped with each spatial grid and aggregated using overall mean for each equity level. We also calculated the local Moran's I for each $3 \mathrm{~km}$ grid to statistically assess how well the high and low EVCS placement densities varied spatially with existing socioeconomic inequalities.

\section{Results}

\subsection{Feature extraction from preprocessed data}

In total we calculated 28 features from the four sources listed in Table 2 for each grid resolution by summarizing the variables using mean and standard deviation for each spatial grid. These features were used as predictor variables to train the machine learning models. The KDE of the existing EVCS placements are shown in Figure 3. These are categorized into three classes low, medium and high and overlaid with the spatial grids at each resolution and used as the response variable for training machine learning models. 


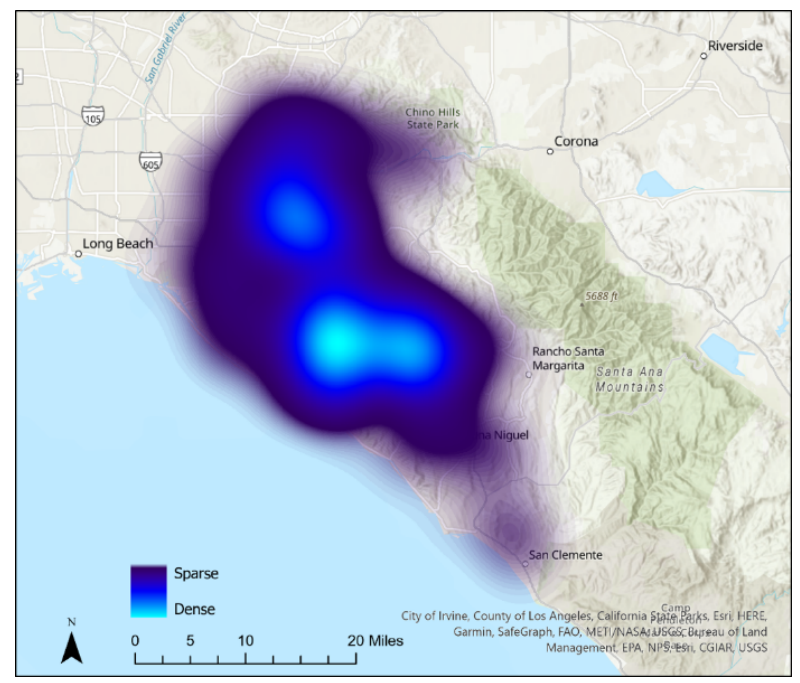

Figure 3: A heatmap showing the kernel density estimate of existing electric vehicle charging station placements in Orange County, California

\subsection{Model accuracy assessment by varying spatial scale}

The cross-validation accuracy of the machine learning models varied based on the spatial grid sizes and training methods. Table 3 lists the 10-fold cross validation accuracy with 3 repeats for random forests, multinomial logit and support vector machines for varying grid sizes from $1 \mathrm{~km}$ to $5 \mathrm{~km}$. Random Forests were the most accurate models with accuracy as high as $94.9 \%$ for the $3 \mathrm{~km}$ spatial grids. Support Vector Machines had an accuracy of $94.8 \%$ followed by multinomial logit with an accuracy of $92.1 \%$ for the $3 \mathrm{~km}$ grids. We chose random forests as the best model as the variability in accuracy scores were the least for random forests across different grid sizes as highlighted by the boxplots in Figure 4.

Table 3: Mean cross-validation accuracy scores of different models with varying spatial resolution

\begin{tabular}{lccc}
\hline Grid Size & $\begin{array}{c}\text { Random } \\
\text { Forest }\end{array}$ & Multinomial Logit Regression & SVM Linear \\
\hline $1 \times 1 \mathrm{~km}$ & 0.9281 & 0.9230 & 0.9260 \\
$2 \times 2 \mathrm{~km}$ & 0.9297 & 0.9182 & 0.9394 \\
$3 \times 3 \mathrm{~km}$ & 0.9499 & 0.9219 & 0.9487 \\
$4 \times 4 \mathrm{~km}$ & 0.9208 & 0.9146 & 0.9479 \\
$5 \times 5 \mathrm{~km}$ & 0.8930 & 0.7920 & 0.8807 \\
\hline
\end{tabular}




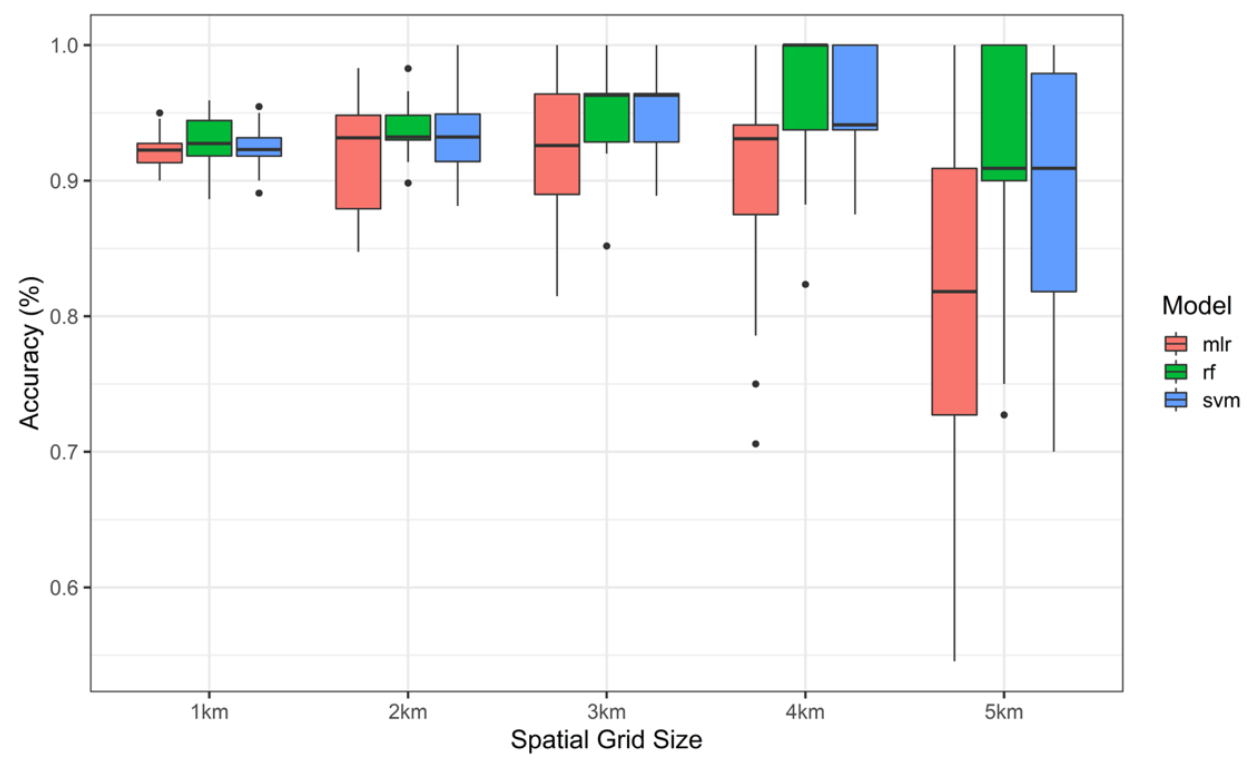

Figure 4: Prediction accuracy of Random Forest, Multinomial Logit and Support vector Machines at varying spatial resolutions

The Random Forest model outperformed both Support Vector Machines and Multinomial Logit in terms of mean cross-validation accuracy for the grids ranging from $1 \mathrm{~km}$ to $3 \mathrm{~km}$. The models become more uncertain in their predictions as we kept increasing the grid size and the variance in the accuracy became large for grid sizes $4 \mathrm{~km}$ and $5 \mathrm{~km}$. The underlying socioeconomic factors were not sufficient to predict placements at these scales as the populations modeled became more heterogeneous as we increased the resolution of our analysis.

\subsection{Confusion matrices for EVCS placement predictions}

The random forests at $3 \mathrm{~km}$ grid sizes had the highest accuracy with lowest variance (Figure 4) and were chosen as the best model for EVCS placement predictions. Additionally, we generated confusion matrices (Figure 5) to distinguish the misclassification rates among EVCS placement density classes across the five grid sizes using Random Forests to further gauge the class level accuracy in predictions as the spatial scale varied. 

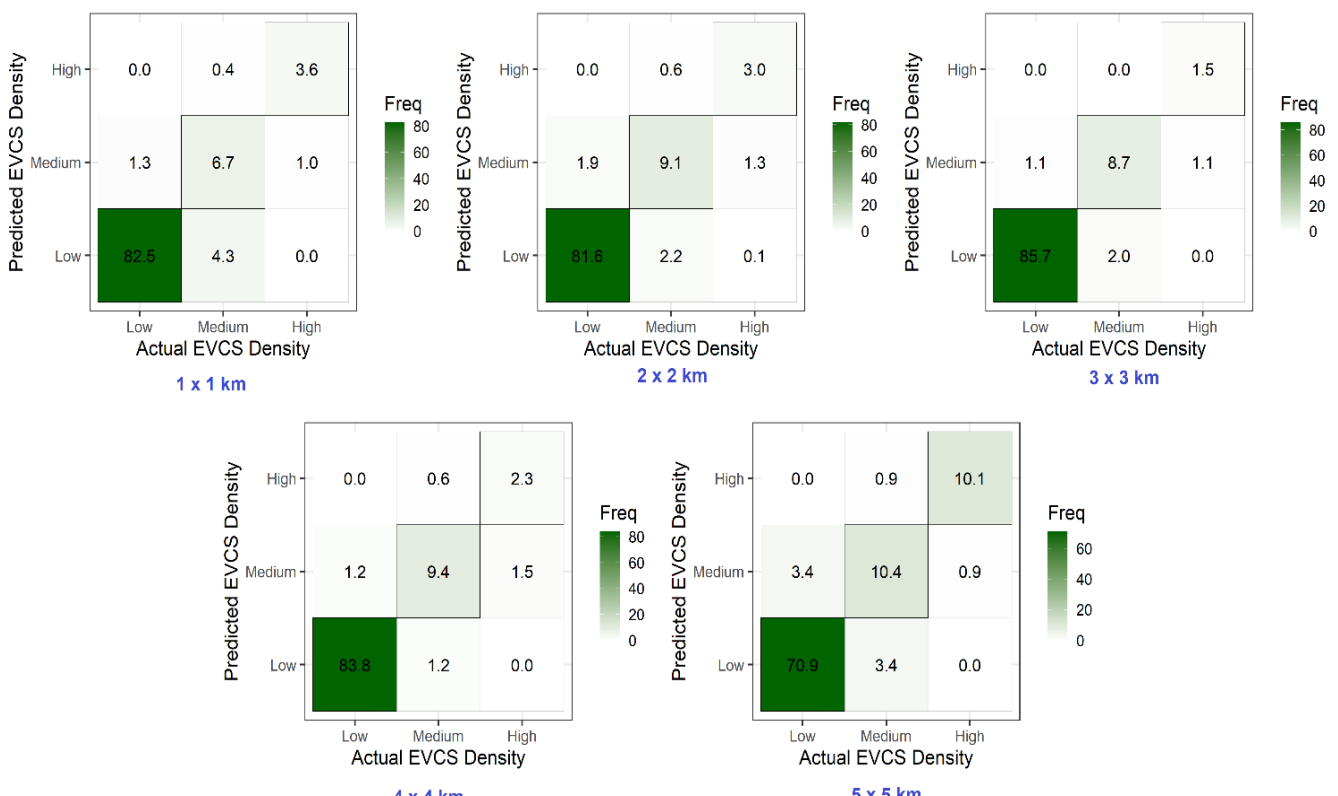

$4 \times 4 \mathrm{~km}$

$5 \times 5 \mathrm{~km}$

Figure 5: Confusion matrices of Random Forest models at varying spatial resolutions for Orange County, CA.

The 'Low' density EVCS placements are most accurately classified for $3 \mathrm{~km}$ grids, whereas the 'Medium' and 'High' density EVCS placements are most accurately classified at the $5 \mathrm{~km}$ resolution, however, these results have a high variance as the uncertainties around the predictions increase as we increase the grid sizes as well. Hence, we chose the $3 \mathrm{~km}$ grids as the most optimal scale for our analysis. Based on existing data the highest number of grids were pre-labelled as low density areas - but a resampling technique was used to reduce overfitting of the models.

\subsection{Examining spatial equity levels of predicted EVCS placements for Orange County}

We examined the spatial accessibility of predicted EVCS placements throughout Orange County using the weighted equity index and categorized the region into three classes based on low, medium and high level of inequities that exist in each $3 \mathrm{~km}$ grid. The spatial inequality index demonstrated areas with low vehicle ownership, high population density, high percentage of 
minority population, high percentage of people living below poverty levels, with less access to education, low median household income were categorized as high inequity areas.

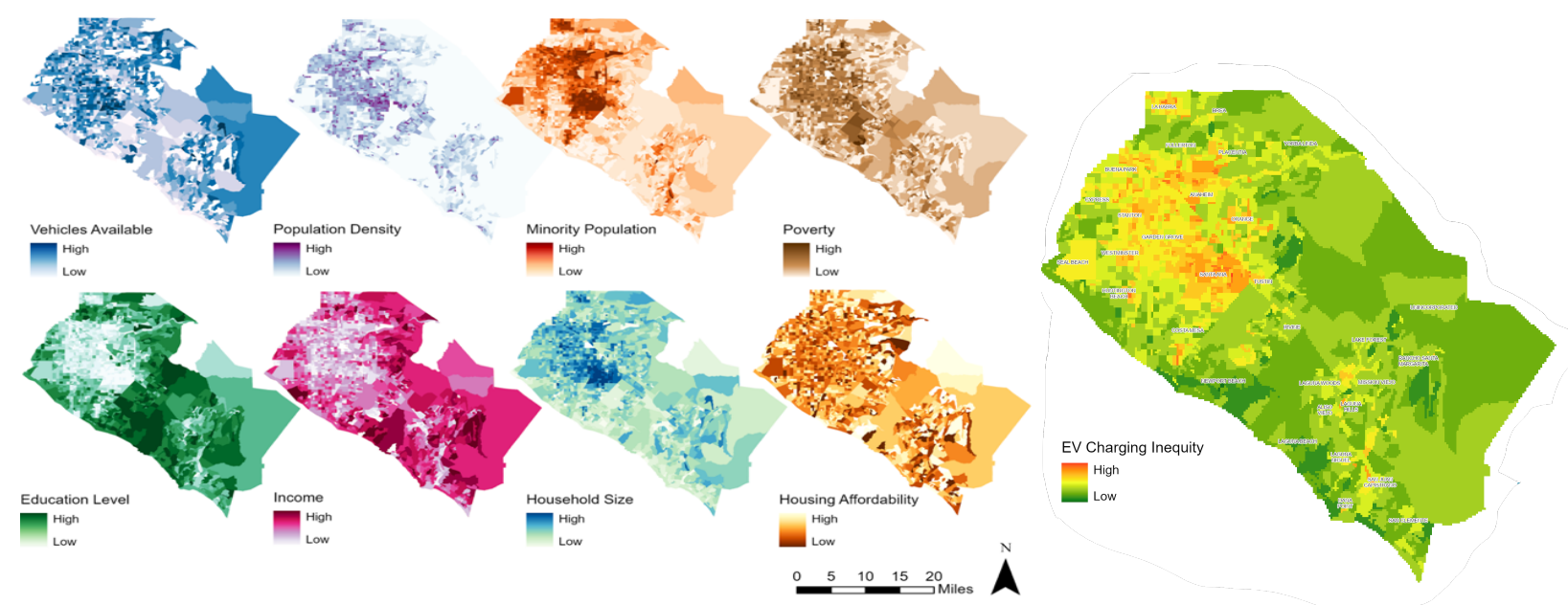

Figure 6: Spatial distribution of socioeconomic composition of populations residing in Orange County along with the weighted equity index showing three levels of spatial inequity in EVCS placements

By mapping out the equity indicators (Fig. 6), it was further revealed that the central part of Orange County near the cities of Santa Ana, some parts of Costa Mesa and Garden Grove appear to have a highest inequity in EVCS access compared to the rest of the county. Cities like Newport Beach, Irvine and Huntington Beach experienced very low inequities as the underlying populations are homogeneous in terms of income levels, education levels as well as vehicle access.

We also estimated generated maps for predicted EVCS placements along with the weighted equity indicator (Figure 7) and computed the percentage of spatial overlap of the EVCS grids with the categorized equity classes from Table 4 . 
Table 4 - Spatial Inequality Index levels of spatial grids in Orange County with respect to socioeconomic and demographic characteristics

\begin{tabular}{|c|c|c|c|c|c|c|c|c|c|c|}
\hline Level & 1 & 2 & 3 & 4 & 5 & 6 & 7 & 8 & 9 & 10 \\
\hline Categories & \multicolumn{3}{|c|}{ High inequity } & \multicolumn{4}{|c|}{ Medium inequity } & \multicolumn{3}{|c|}{ Low inequity } \\
\hline $\begin{array}{l}\text { Vehicles } \\
\text { Available } \\
\text { (per } \\
\text { household) }\end{array}$ & $\begin{array}{l}0.58- \\
1.17\end{array}$ & $\begin{array}{l}1.18- \\
1.46\end{array}$ & $\begin{array}{l}1.47- \\
1.69\end{array}$ & $\begin{array}{l}1.70- \\
1.88\end{array}$ & $\begin{array}{l}1.89- \\
2.05\end{array}$ & $\begin{array}{l}2.06- \\
2.21\end{array}$ & $\begin{array}{l}2.22- \\
2.39\end{array}$ & $\begin{array}{l}2.40- \\
2.60\end{array}$ & $\begin{array}{l}2.61- \\
2.95\end{array}$ & $\begin{array}{l}2.96- \\
4.17\end{array}$ \\
\hline $\begin{array}{l}\text { Population } \\
\text { Density (Per } \\
\text { square mile) }\end{array}$ & $\begin{array}{l}19371 \\
- \\
35671 \\
\end{array}$ & $\begin{array}{l}12255 \\
- \\
19370\end{array}$ & $\begin{array}{l}8588 \\
- \\
12254 \\
\end{array}$ & $\begin{array}{l}6463- \\
8587\end{array}$ & $\begin{array}{l}5031 \\
- \\
6462 \\
\end{array}$ & $\begin{array}{l}3924- \\
5030\end{array}$ & $\begin{array}{l}2979- \\
3923\end{array}$ & $\begin{array}{l}2120- \\
2978\end{array}$ & $\begin{array}{l}1193- \\
2119\end{array}$ & $\begin{array}{l}0- \\
1192\end{array}$ \\
\hline $\begin{array}{l}\text { Underreprese } \\
\text { nted Minority } \\
\text { Population } \\
(\%)\end{array}$ & $\begin{array}{l}0.86- \\
1\end{array}$ & $\begin{array}{l}0.73- \\
0.85\end{array}$ & $\begin{array}{l}0.60- \\
0.72\end{array}$ & $\begin{array}{l}0.49- \\
0.59\end{array}$ & $\begin{array}{l}0.39- \\
0.48\end{array}$ & $\begin{array}{l}0.31- \\
0.38\end{array}$ & $\begin{array}{l}0.23- \\
0.30\end{array}$ & $\begin{array}{l}0.17- \\
0.22\end{array}$ & $\begin{array}{l}0.10- \\
0.16\end{array}$ & $\begin{array}{l}0- \\
0.09\end{array}$ \\
\hline $\begin{array}{l}\text { Population } \\
\text { Below } \\
\text { Poverty (\%) }\end{array}$ & $\begin{array}{l}0.48- \\
0.82\end{array}$ & $\begin{array}{l}0.36- \\
0.47\end{array}$ & $\begin{array}{l}0.27- \\
0.35\end{array}$ & $\begin{array}{l}0.21- \\
0.26\end{array}$ & $\begin{array}{l}0.16- \\
0.20\end{array}$ & $\begin{array}{l}0.12- \\
0.15\end{array}$ & $\begin{array}{l}0.08- \\
0.11\end{array}$ & $\begin{array}{l}0.05- \\
0.07\end{array}$ & $\begin{array}{l}0.03- \\
0.05\end{array}$ & $\begin{array}{l}0- \\
0.02\end{array}$ \\
\hline $\begin{array}{l}\text { Education } \\
\text { Level } \\
\text { (Bachelor's } \\
\text { or higher for } \\
\text { pop over 25) }\end{array}$ & $\begin{array}{l}0- \\
0.11\end{array}$ & $\begin{array}{l}0.12- \\
0.19\end{array}$ & $\begin{array}{l}0.20- \\
0.26\end{array}$ & $\begin{array}{l}0.27- \\
0.34\end{array}$ & $\begin{array}{l}0.35- \\
0.41\end{array}$ & $\begin{array}{l}0.42- \\
0.48\end{array}$ & $\begin{array}{l}0.49- \\
0.55\end{array}$ & $\begin{array}{l}0.56- \\
0.63\end{array}$ & $\begin{array}{l}0.64- \\
0.73\end{array}$ & $\begin{array}{l}0.74- \\
1\end{array}$ \\
\hline $\begin{array}{l}\text { Median } \\
\text { Household } \\
\text { Income (\$) }\end{array}$ & $\begin{array}{l}15444 \\
- \\
44063\end{array}$ & $\begin{array}{l}44064 \\
- \\
59141\end{array}$ & $\begin{array}{l}59141 \\
- \\
72888\end{array}$ & $\begin{array}{l}72889 \\
- \\
87589\end{array}$ & $\begin{array}{l}87560 \\
- \\
10280 \\
3 \\
\end{array}$ & $\begin{array}{l}10280 \\
4- \\
12017 \\
9 \\
\end{array}$ & $\begin{array}{l}120180 \\
- \\
141429\end{array}$ & $\begin{array}{l}14143 \\
0- \\
16947 \\
9 \\
\end{array}$ & $\begin{array}{l}16948 \\
0- \\
21022 \\
1 \\
\end{array}$ & $\begin{array}{l}21022 \\
2- \\
25000 \\
1\end{array}$ \\
\hline $\begin{array}{l}\text { Household } \\
\text { Size }\end{array}$ & $\begin{array}{l}5.04- \\
7.51\end{array}$ & $\begin{array}{l}4.30- \\
5.03\end{array}$ & $\begin{array}{l}3.82- \\
4.29\end{array}$ & $\begin{array}{l}3.44- \\
3.81\end{array}$ & $\begin{array}{l}3.13- \\
3.43\end{array}$ & $\begin{array}{l}2.87- \\
3.12\end{array}$ & $\begin{array}{l}2.58- \\
2.86\end{array}$ & $\begin{array}{l}2.22- \\
2.57\end{array}$ & $\begin{array}{l}1.76- \\
2.21\end{array}$ & $\begin{array}{l}1.00- \\
1.75\end{array}$ \\
\hline $\begin{array}{l}\text { Housing } \\
\text { Affordability, } \\
\text { pop Spending } \\
>30 \% \text { of } \\
\text { income (\%) }\end{array}$ & $\begin{array}{l}0.91- \\
1\end{array}$ & $\begin{array}{l}0.78- \\
0.90\end{array}$ & $\begin{array}{l}0.69- \\
0.77\end{array}$ & $\begin{array}{l}0.61- \\
0.68\end{array}$ & $\begin{array}{l}0.53- \\
0.60\end{array}$ & $\begin{array}{l}0.46- \\
0.52\end{array}$ & $\begin{array}{l}0.36- \\
0.45\end{array}$ & $\begin{array}{l}0.25- \\
0.35\end{array}$ & $\begin{array}{l}0.10- \\
0.24\end{array}$ & $\begin{array}{l}0- \\
0.09\end{array}$ \\
\hline
\end{tabular}




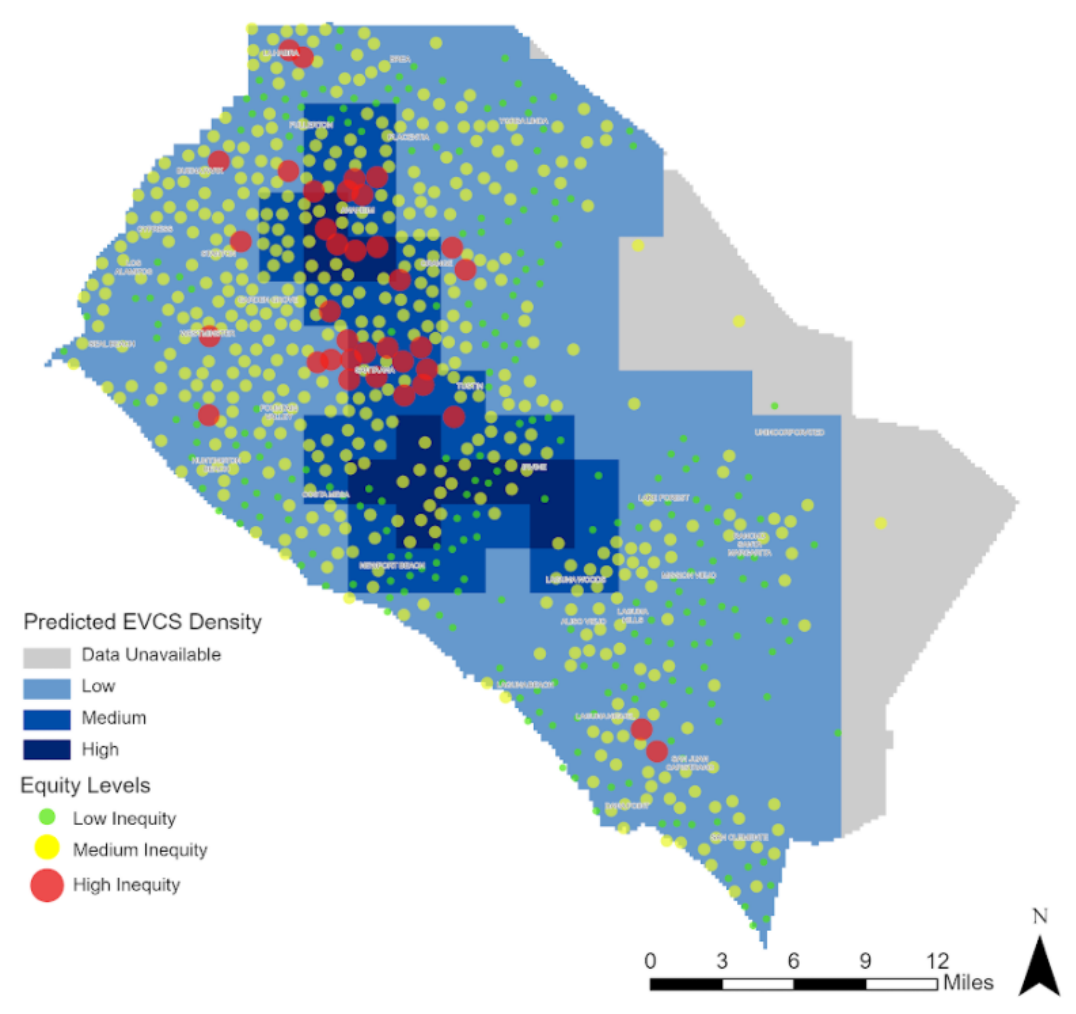

Figure 7: Map showing the predicted EVCS placements in Orange County along with spatial inequity levels.

We found that $50.32 \%$ of the area in Orange County that were categorized as high inequity regions would see a medium density of predicted EVCS placements, whereas only $9 \%$ of these regions would see a high density of EVCS placements. The low inequity areas would experience very few EVCS placements with only $1.56 \%$ of medium density placements and $0.78 \%$ of highdensity EVCS placements.

Table 5 - Proportion of spatial coverage of predicted EVCS placements categorized by equity levels

\begin{tabular}{|c|c|c|c|}
\hline \multirow{2}{*}{$\begin{array}{c}\text { Predicted EVCS } \\
\text { Placements Density }\end{array}$} & High inequity & Medium inequity & Low inequity \\
\cline { 2 - 4 } & $14.86 \%$ & $2.61 \%$ & $0 \%$ \\
\hline Low & $50.32 \%$ & $9.92 \%$ & $1.56 \%$ \\
\hline Medium & $9.00 \%$ & $5.35 \%$ & $0.78 \%$ \\
\hline
\end{tabular}


The central part of Orange County still shows a high density of future EVCS placements based on our predictions. However, the small pockets of high inequity regions within the central part of the county do seem to have a medium density of predicted EVCS placements. The area with high inequity in the southern parts of the county continues to show a low density of EVCS placements based on the socioeconomic factors used in our model predictions.

\section{Discussion}

Our study incorporates machine learning along with spatial accessibility measurement to highlight the spatial disparities in EVCS placements based on the socioeconomic composition of cities in Orange County, California. Since the methodology developed in this study uses a datadriven approach, the model performance and predictions depend upon the availability of good quality data. The spatial inequality indicator (Figure 6) was derived by combining multiple factors at play like - vehicle ownership, poverty levels, educational access, income levels and housing affordability (Table 4) which was a step forward in terms of capturing some of the complex interactions among the underlying social, economic and demographic characteristics at play.

The random forest algorithm reaches the highest predictive accuracy for the models compared to support vector machines and multinomial logit (Table 3). The model accuracies are also affected by the scale of analysis as shown in Figure 4. The accuracy of the models keep increasing as we increase the resolution from $1 \mathrm{~km}$ up to $3 \mathrm{~km}$ but sharply declines after that. A possible reason could be that the uncertainties in prediction amplify as we make the spatial units of analysis much larger and there is more spatial heterogeneity at scales of $4 \mathrm{~km}$ or above which are not captured completely by the models. The current study also limits us in terms of EVCS data as we have a sparse coverage of existing EVCS locations on the southeast corner of the county and large 
portions of the eastern part also do not have any ground truth to compare model predictions with. But it is quite evident that the optimal choice of the grid size can distinctively change the predictive accuracy of the models. Hence, this study further validates the fact that scale effects in transportation planning is crucial in determining service areas and choosing suitable sites for future infrastructure investments.

Better decision-making on infrastructure allocation could be facilitated by our conceptual framework. As shown by our results, the spatial inequity measurement can be summarized into three different categories namely - high inequity, medium inequity and low inequity. Considering that people in low income densely populated neighborhoods with low job accessibility tend to have least access to public EV charging stations, it is recommended to place new and supplementary EV charging stations with extended operating hours in the central part of the Orange County area. The cold spots which have lower inequity levels could be less prioritized, given that they are located in green belts and have lower population density.

The framework developed in this study is generalizable and can be applied to any type of study area. With the improved availability of sufficient amount of data with high spatial and temporal granularity the inequity index can be expanded to include diverse infrastructure, such as healthcare resources (Chen et al. 2020, Wang et al. 2020) and food outlets (Chen et al. 2017, Wang et al. 2018). The inequity levels help in identifying a particular region within the study area that has limited access to EVCS and proposing locations to place additional EV charging infrastructure.

\section{Conclusion}

In this work, we presented a machine learning framework for the prediction of EV charging station placements to address issues of spatial accessibility. Unlike previous work, we utilized 
social, economic, and demographic data along with the historical charging data to characterize future placements of new EV charging infrastructure. We trained three popular machine learning models along with social inequity levels at various spatial scales for the prediction of charging stations placements. The results obtained in terms of prediction performance is superior to the results in the previous works. Although several existing approaches address cost benefits to optimize EV charging access, out study is one of the first to address and account for spatial inequality to EVCS placements by combining socioeconomic characteristics of underlying populations in multiple cities within a study area.

Our framework can be improved further by including additional measures that contribute to low spatial accessibility like job density and updated EVCS density data from future counts conducted by federal agencies. The scale effects can also be further tuned by testing model behavior at finer spatial grids based upon the availability of more ground truth data on EV charging stations.

\section{Acknowledgments}

The authors would like to thank the Department of Urban Planning and Public Policy at the University of California, Irvine for providing support for this research.

\section{Author Contributions}

ML did data collection, modeling, and analysis. Research design, visualization, interpretation of the results, and manuscript preparation were conducted by AR. 


\section{References}

1. Ahmad, F., Iqbal, A., Ashraf, I., \& Marzband, M. (2022). Optimal location of electric vehicle charging station and its impact on distribution network: A review. Energy Reports, 8, 2314-2333.

2. Almaghrebi, A., Aljuheshi, F., Rafaie, M., James, K., \& Alahmad, M. (2020). Data-driven charging demand prediction at public charging stations using supervised machine learning regression methods. Energies, 13(16), 4231.

3. Bakker, Sjoerd, and Jan Jacob Trip. "Policy options to support the adoption of electric vehicles in the urban environment." Transportation Research Part D: Transport and Environment 25 (2013): 18-23.

4. Bansal, Prateek, Kara M. Kockelman, and Yiyi Wang. "Hybrid electric vehicle ownership and fuel economy across Texas: an application of spatial models." Transportation Research Record 2495, no. 1 (2015): 53-64.

5. Brady, John, and Margaret O’Mahony. "Travel to work in Dublin. The potential impacts of electric vehicles on climate change and urban air quality." Transportation Research Part D: Transport and Environment 16, no. 2 (2011): 188-193.

6. Canepa, Kathryn, Scott Hardman, and Gil Tal. "An early look at plug-in electric vehicle adoption in disadvantaged communities in California." Transport Policy 78 (2019): 19-30.

7. Chen, B.Y., et al., 2017. Measuring place-based accessibility under travel time uncertainty. International Journal of Geographical Information Science, 31 (4), 783-804. doi:10.1080/ 13658816.2016.1238919.

8. Chen, B.Y., et al., 2020. Evaluating spatial accessibility to healthcare services under travel time uncertainty: a reliability-based floating catchment area approach. Journal of Transport Geography, 87, 102794. doi:10.1016/j.jtrangeo.2020.102794

9. Dalton, A. M., Jones, A., Ogilvie, D., Petticrew, M., White, M., \& Cummins, S. (2013). Using spatial equity analysis in the process evaluation of environmental interventions to tackle obesity: the healthy towns programme in England. International journal for equity in health, 12(1), 1-10.

10. Dong, Guanpeng, Jing Ma, Ran Wei, and Jonathan Haycox. "Electric vehicle charging point placement optimisation by exploiting spatial statistics and maximal coverage location models." Transportation Research Part D: Transport and Environment 67 (2019): 77-88. 
11. Egbue, Ona, and Suzanna Long. "Barriers to widespread adoption of electric vehicles: An analysis of consumer attitudes and perceptions." Energy policy 48 (2012): 717-729.

12. Farkas, Z., Shin, H. S., \& Nickkar, A. (2018). Environmental attributes of electric vehicle ownership and commuting behavior in maryland: Public policy and equity considerations. Mid-Atlantic Transportation Sustainability University Transportation Center. Retrieved April, 20, 2019.

13. Fleming, K. L. (2018). Social equity considerations in the new age of transportation: Electric, automated, and shared mobility. Journal of Science Policy \& Governance, 13(1), 20.

14. Guo, Shuocheng, and Eleftheria Kontou. "Disparities and equity issues in electric vehicles rebate allocation." Energy Policy 154 (2021): 112291.

15. Hardman, S., Fleming, K. L., Khare, E., \& Ramadan, M. M. (2021). A perspective on equity in the transition to electric vehicles.

16. Hawkins, Troy R., Bhawna Singh, Guillaume Majeau-Bettez, and Anders Hammer Strømman. "Comparative environmental life cycle assessment of conventional and electric vehicles." Journal of industrial ecology 17, no. 1 (2013): 53-64.

17. Hsu, Chih-Wei, and Kevin Fingerman. "Public electric vehicle charger access disparities across race and income in California." Transport Policy 100 (2021): 59-67.

18. Ju, Y., Cushing, L. J., \& Morello-Frosch, R. (2020). An equity analysis of clean vehicle rebate programs in California. Climatic Change, 162(4), 2087-2105.

19. Ku, A., Kammen, D. M., \& Castellanos, S. (2021). A quantitative, equitable framework for urban transportation electrification: Oakland, California as a mobility model of climate justice. Sustainable Cities and Society, 74, 103179.

20. Law, M., \& Roy, A. (2021, November). A geospatial data fusion framework to quantify variations in electric vehicle charging demand. In Proceedings of the 4th ACM SIGSPATIAL International Workshop on Advances in Resilient and Intelligent Cities (pp. 23-26).

21. Li, G., Luo, T., \& Song, Y. (2022). Spatial equity analysis of urban public services for electric vehicle chargingImplications of Chinese cities. Sustainable Cities and Society, 76, 103519. 
22. Li, G., Luo, T., \& Song, Y. (2022). Spatial equity analysis of urban public services for electric vehicle chargingImplications of Chinese cities. Sustainable Cities and Society, 76, 103519.

23. Marcantonio, R. A., Golub, A., Karner, A., \& Nelson Dyhle, L. (2017). Confronting inequality in metropolitan regions: Realizing the promise of civil rights and environmental justice in metropolitan transportation planning. Fordham Urb. LJ, 44, 1017.

24. Roy, A., \& Kar, B. (2022). A multicriteria decision analysis framework to measure equitable healthcare access during COVID-19. Journal of transport \& health, 101331.

25. She, Zhen-Yu, Qing Sun, Jia-Jun Ma, and Bai-Chen Xie. "What are the barriers to widespread adoption of battery electric vehicles? A survey of public perception in Tianjin, China." Transport Policy 56 (2017): 29-40.

26. Sheldon, T. L. (2022). Evaluating Electric Vehicle Policy Effectiveness and Equity. Annual Review of Resource Economics, 14.

27. Shibl, M., Ismail, L., \& Massoud, A. (2020). Machine learning-based management of electric vehicles charging: Towards highly-dispersed fast chargers. Energies, 13(20), 5429.

28. The United States Government. (2021, December 13). Fact sheet: The biden-harris electric vehicle charging action plan. The White House. Retrieved December 22, 2021, from https://www.whitehouse.gov/briefingroom/statements-releases/2021/12/13/fact-sheet-the-biden-harris-electric-vehicle-charging-action-plan/ utility perspective. Journal of Transport Geography, 73, 13-24. doi:10.1016/j.jtrangeo.2018.10.002

29. Wang, J., et al., 2020. Access to hospitals: potential vs. observed. Cities, 100, 102671. doi:10.1016/j. cities.2020.102671

30. Wang, Y., et al., 2018. Measuring temporal variation of location-based accessibility using space-time

31. Williams, James H., Andrew DeBenedictis, Rebecca Ghanadan, Amber Mahone, Jack Moore, William R. Morrow, Snuller Price, and Margaret S. Torn. "The technology path to deep greenhouse gas emissions cuts by 2050: the pivotal role of electricity." science 335, no. 6064 (2012): 53-59.

32. Ye, B., Jiang, J., Miao, L., Yang, P., Li, J., \& Shen, B. (2015). Feasibility study of a solar-powered electric vehicle charging station model. Energies, 8(11), 13265-13283. 